\title{
Preliminary Numerical Research of Microstructural Fracture Behavior in Metal by Using Interface Element*
}

\author{
by Seigo Tomiyama**, Hisahsi Serizawa***, Tsuyoshi Hajima** and Hidekazu Murakawa***
}

\begin{abstract}
In order to demonstrate not only the deformation of grain but also the opening and/or sliding at grain boundary, the interface element was introduced into the ordinary finite element method, and this numerical method was applied for examining the microstructural fracture behavior in two-dimensional ideal microstructure obtained through Voronoi tessellations. As for the grain, the anisotropy in elastic modulus due to the grain orientation was taken into account, while the fracture strength at grain boundary was assumed to be related to the boundary energy which could be determined by the atomic disorder at the boundary. From the serial computational results for examining the influences of elastic properties in grain (isotropy and anisotropy), mechanical property at grain boundary (interaction between opening and sliding deformation), and grain configurations, it was revealed that all the factors varied in this research might affect the microstructural fracture behavior. Also, it can be concluded that this numerical method with the interface element can be useful for demonstrating the microstructural fracture behavior including the deformation at grain boundary.
\end{abstract}

Key Words: Interface Element, Grain Orientation, Grain Boundary, Finite Element Method, Stress Concentration

\section{Introduction}

Recently, super high strength steel has been developed by controlling its microstructure precisely and this steel has been widely used in various fields. Although there have been various experimental studies about fracture behavior of the high strength steel, its microstructural deformations have not been revealed because only the deformation of grain has been modeled in the conventional finite element method. The authors have developed the interface element in order to model the interfacial deformation such as crack opening, crack propagation and interfacial sliding ${ }^{1)}$. In this research, the interface element was employed for modeling the deformation of grain boundary and the microstructural fracture behavior was examined by using the finite element method with the interface element. Namely, the anisotropic deformation of grain due to grain orientation was modeled by the ordinary finite element and only elastic deformation of grain was considered as the preliminary numerical examination. On the other hand, the opening and sliding at grain boundary was demonstrated by the interface element where the effect of grain orientation on the mechanical property at the grain boundary was taken into account. By using two dimensional microstructure obtained through Voronoi tessellations, the influence of grain orientation on the microstructural fracture behavior was investigated.

\footnotetext{
*Received: 2010.11.11

${ }^{* *}$ Student Member, Graduate School of Engineering, Osaka University

${ }^{* * *}$ Member, Joining and Welding Research Institute, Osaka University
}

\section{Analysis Method}

\subsection{Model for analysis}

Two dimensional ideal microstructures of steel were created by using Voronoi tessellations and Fig. 1 shows two kinds of finite element models based on these microstructures whose size was $426 \times 426 \mu \mathrm{m}^{2}$. These models contain 30 grains, whose orientations are varied. In order to demonstrate the interfacial deformation, the interface elements were arranged along the grain boundaries.

\subsection{Interface element}

Essentially, the interface element is the distributed nonlinear spring existing between surfaces forming the interface or the potential crack surfaces. The interaction between surfaces is characterized by a potential function that involves the surface energy $\gamma$, the shape parameter $N$ and the scale parameter $r_{0}$. In order to describe both the opening and sliding deformations at the grain boundary, the combined potential function was employed in

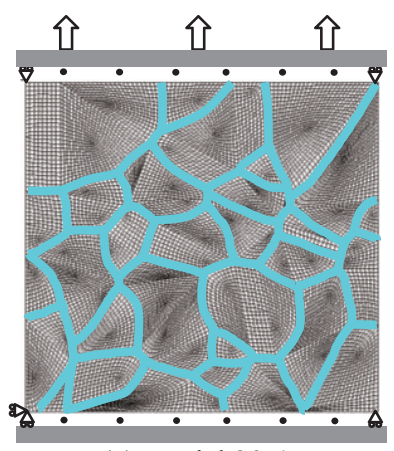

(a) Model 30-1

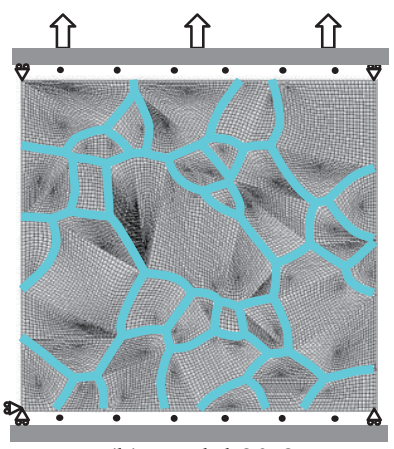

(b) Model 30-2
Fig. 1 Finite element models for analysis and boundary conditions. 


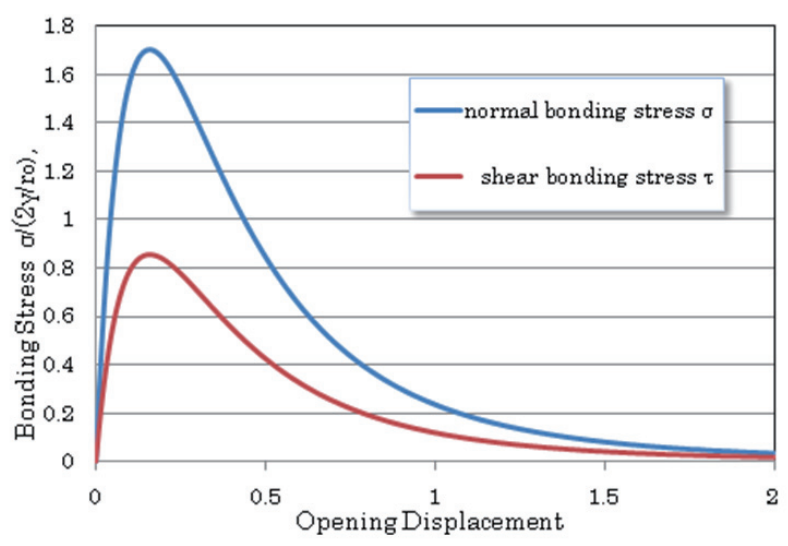

Fig. 2 Opening and shear stresses related to displacement.

Table 1 Stiffness of hexagonal crystal for $a$-Fe.

\begin{tabular}{|c|ccr|}
\hline hexagonal & \multicolumn{3}{|c|}{$\left(10^{-11} \mathrm{~Pa}^{-1}\right)$} \\
\cline { 2 - 4 } crystal & $S_{11}$ & $S_{12}$ & $S_{44}$ \\
\hline & 0.80 & -0.28 & 0.86 \\
\hline
\end{tabular}

this research $^{1)}$. The opening and shear stresses to the displacement $\delta$ could be described as the following equations, and these relationships are shown in Fig. 2.

$$
\begin{aligned}
& \sigma=\frac{4 \gamma N}{r_{0}}\left\{\left(\frac{r_{0}}{r_{0}+\delta}\right)^{N+1}-\left(\frac{r_{0}}{r_{0}+\delta}\right)^{2 N+1}\right\} \\
& \tau=\frac{4 \sqrt{A} \gamma N}{r_{0}}\left\{\left(\frac{r_{0}}{r_{0}+\delta}\right)^{N+1}-\left(\frac{r_{0}}{r_{0}+\delta}\right)^{2 N+1}\right\}
\end{aligned}
$$

The parameters involving above equations were determined for demonstrating $\alpha$-Fe fracture behavior. Namely, the surface energy $\gamma$ and the shape parameter $N$ were assumed to $2.0 \mathrm{~N} / \mathrm{mm}$ and 4 , respectively. The scale parameter $r_{0}$ is the property related to maximum bonding and sliding stresses $\left(\sigma_{\max }\right.$ and $\left.\tau_{\max }\right)$, and is determined according to the misorientation angle between neighbor grains as described in the following section. Also, the interaction parameter $A$ which indicates the ratio of $\tau_{\max }$ to $\sigma_{\max }$ is included in Eq. (2).

\subsection{Anisotropy in grains}

The properties of each grain in metal material have anisotropy due to the direction of grain growth. In order to demonstrate the microstructural deformation precisely, the anisotropy has to be taken into account and was assumed by changing the elastic moduli of each direction $\left(E_{11}, E_{22}\right.$ and $\left.G_{12}\right)$. The material studied in this research was $\alpha$-Fe which has hexagonal crystal lattice, and target plane was assumed to (110) plane which contains only tilt boundaries not twist boundaries. Table 1 shows the stiffness of hexagonal crystal for $\alpha-\mathrm{Fe}^{2)}$, and the elastic moduli in anisotropic grain were determined according to the

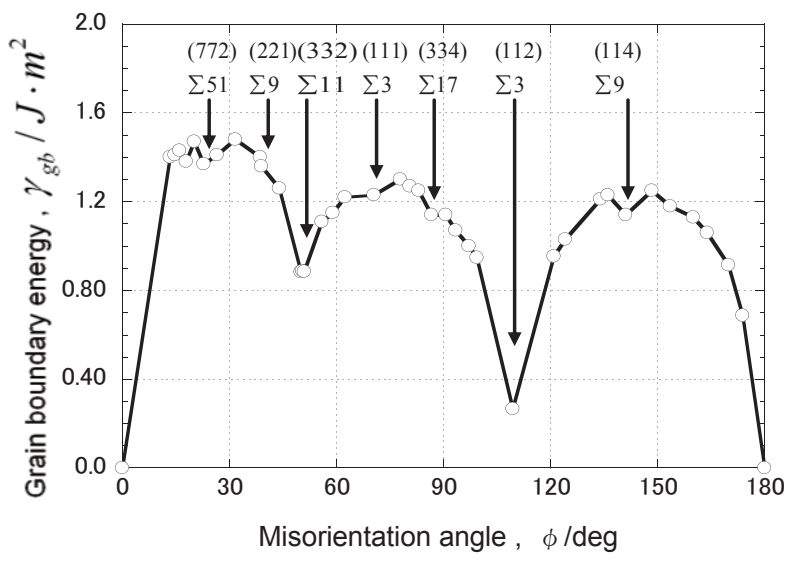

Fig. 3 Relationship between boundary fracture stress and misorientation angle

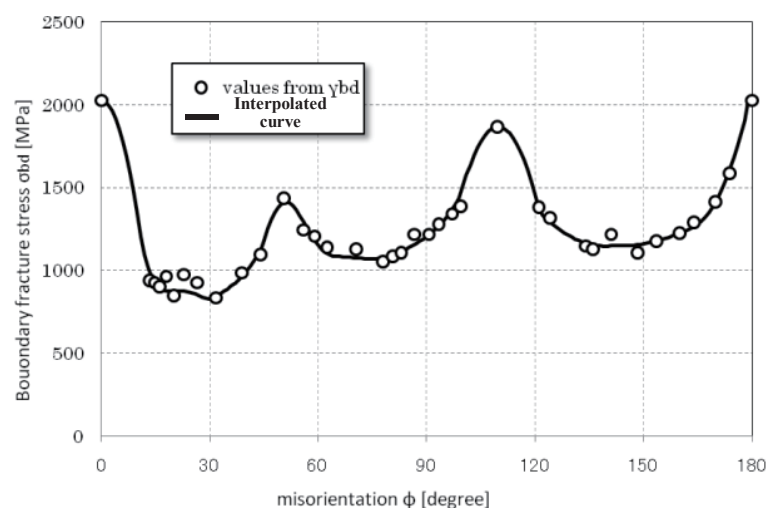

Fig. 4 Relationship between boundary fracture stress and misorientation angle.

direction of target plane. Namely, the moduli of each direction in (110) plane $\left(E_{11}, E_{22}\right.$ and $\left.G_{12}\right)$ were assumed to 125.0, 210.5 and $66.20 \mathrm{GPa}$, respectively.

\subsection{Anisotropy in grain boundary}

Generally, the intergranular fracture is caused by crack opening and sliding at grain boundaries. The interface element can demonstrate such deformations by employing the interaction parameter $A$ in Eq. (2). In the case with small value of parameter $A$, the interfacial sliding easily occurs in comparison with the crack opening at the interface. The parameter $A$ was varied as $1.0,0.25$ and 0.01 in order to examine the influence of difference between opening and shear strength $\left(\sigma_{\max }\right.$ and $\left.\tau_{\max }\right)$ on the microstructural fracture behavior.

The grain boundary can be regarded as disorder of atoms between neighbor grains. So, it can be assumed that the fracture strength at grain boundary would be related to the boundary energy, which could be determined by the atomic disorder at the boundary. There have been many researches about the symmetric tilt boundary, and various boundary energies in different plane were studied by using the molecular dynamic method. Tanaka et al. examined $<110>$ boundary energy of 
molybdenum and revealed that the square of fracture strength $\sigma_{\mathrm{gb}}$ would be linearly proportional to the grain boundary energy $\gamma_{\mathrm{bd}}{ }^{3)}$. Since the boundary energy of $\alpha$-Fe was reported as shown in Fig. $3^{4)}$, the authors assumed that the opening fracture stress of $\alpha-\mathrm{Fe}$ would follow the relation to misorientation as shown in Fig. 4 according to a linear law reported by Tanaka et al.

\section{Results and discussions}

\subsection{Deformations in microstructural model}

The conventional finite element method only demonstrates the change of stress \& strain distributions in the grains of microstructural model, while both the stress \& strain distribution of grains and the opening \& sliding at grain boundaries can be described by the finite element method with interface element. Since each grain was assumed to have another orientation to neighbor grains, the following behavior can be obtained in the computational results by using interface element.

1) Stress concentration due to the difference of elastic property between neighbor grains

2) Various deformations at grain boundaries caused by the various mechanical properties of interface element

Also, these two behaviors are strongly affected by the load direction to the orientations of both grain and grain boundary. So, in order to understand the computational results of the multi-grains models employed in this research, simple examinations should be conducted and be evaluated by using only two and triple grains model. However, as preliminary computational researches of microstructural fracture behavior using the interface element, the deformation of grain and the crack initiation \& growth process at grain boundary in 30 grains models were examined in order to reveal the potentiality of this new numerical method. Especially, the influences of anisotropy in grain, interaction parameter $A$ and grain configuration on microstructural fracture behavior were studied.

\subsection{Influence of anisotropy in grain}

As a preliminary analysis, the influence of anisotropy in grains on fracture behavior of the models shown in Fig. 1(a) was

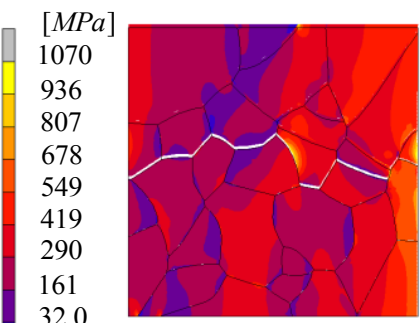

(a) Isotropic model

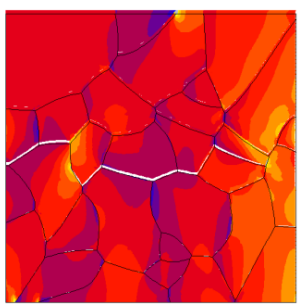

(b) Anisotropic model
Fig. 5 Tensile stress distribution of Model 30-1 $(A=0.25)$. examined with assuming only the elastic deformation in grain while the grain boundary had anisotropy due to the difference of orientation between neighbor grains. Figures 5 shows the typical results of isotropic and anisotropic grains in Model 30-1 under $1.200 \%$ strain, respectively. The interaction parameter $A$ in Eq. (2) was assumed to 0.25 , which means that the shear sliding stress $\tau$ is half of the bonding stress $\sigma$. In the isotropic grain model, the significant difference among grains was not obtained and the stress distribution was almost homogeneous. So, the position of crack propagation was determined by the distribution of boundary fracture stress at grain assumed because of the anisotropy at grain boundary. On the other hand, the stress distribution of anisotropic grain model was heterogeneous and stresses among grains were different. Then, this difference was considered to affect the crack propagation paths as shown in Fig. 5.

\subsection{Influence of interaction parameter $A$}

In order to examine the effect of interaction parameter $A$ on the fracture behavior, the various analyses were conducted by changing the parameter $A$ with assuming the isotropy of grain deformation. The parameter $A$ is related to the relationship between the bonding strength $\sigma$ and the shear strength $\tau$ at the grain boundary, and a small value of $A$ means that the interfacial sliding is easy to occur as compared with the crack opening at grain boundary. Although there would be no experimental results this relationship, the parameter $A$ was assumed to be smaller than 1.0 since the interfacial sliding was considered to start against the crack opening at the grain boundary. In this research, all the boundaries were assumed to have same value of $A$ though the critical opening and sliding strength at grain boundary would be controlled by the microstructure of boundary. So each boundary was defined to have different opening and shear strength depending on the misorientation angles of neighbor grains.

Figure 6 shows the computed results of isotropic Model 30-1 in applying $3.00 \%$ strain and the initiation behaviors of cracks. As the values of parameter $A, 1.0,0.25$ and 0.01 were selected and these means that the shear strength $\tau$ was same, half and one tenth of the opening strength, respectively. Model 30-1 consists of 30 grains and includes 72 types of grain boundaries since each grain was assumed to have different orientation. So, the bonding (opening) strength in Model 30-1 varies from 826 to $1856 \mathrm{MPa}$. However, the opening strength at the initial fracture boundaries shown in Fig. 6 was less than $1242 \mathrm{MPa}$ in all cases. This behavior seems to indicate that the crack opening would not be directly affect by the opening fracture strength at the grain boundary and an external restrain caused by neighbor grains 


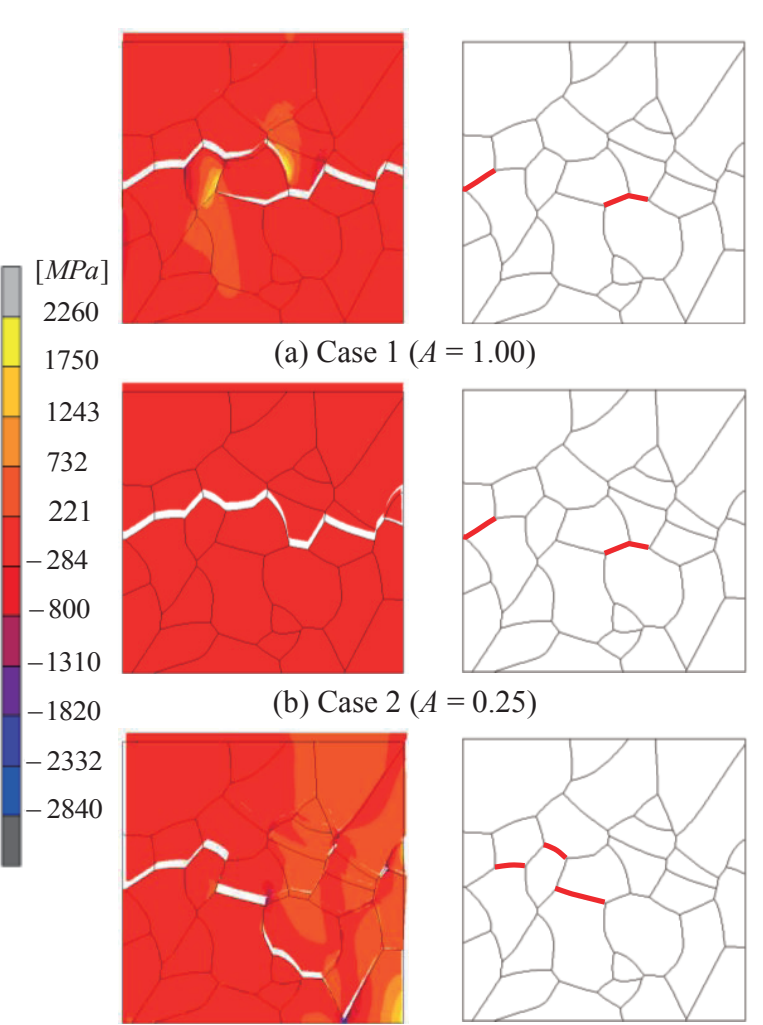

(c) Case $3(A=0.01)$

Fig. 6 Fracture patterns and initiations of crack propagations.

might influence the crack initiation behavior. In other words, the grain boundary with minimum opening fracture strength might not become the initial fracture point.

As shown in Figs. 6(a) and (b), the crack paths of Case 1 and 2 were different though the initial cracks were same. From the precise examination of fracture behaviors in Case 1 and 2, it was found that one of the initial cracks closed after proceeding the fracture process in Case 2 due to opening at neighbor grain boundary. In the current model for interface element, the stiffness of interface element was controlled by the displacement at grain boundary without any decision of fracture. So, there would be a problem that the stiffness of interface element which already opened might sometimes recover because the other neighbor interface element opened and the displacement of the initial crack apparently became smaller. As a future works, this problem has to be fixed in order to examine the microstructural fracture mechanism by using the interface element.

On the other hand, both the initial cracks and the crack path in Case 3 were quite different from those in other cases. In Case 3, many shear deformations at grain boundary were obtained where the sliding direction at grain boundary was almost parallel to the load direction. So the difference in fracture behavior between Case 3 and other cases was consider to be caused by the occurrence of interfacial sliding deformation. Namely, it can be concluded that the interaction parameter $A$ would affect the microstructural fracture behavior when it has a small value.

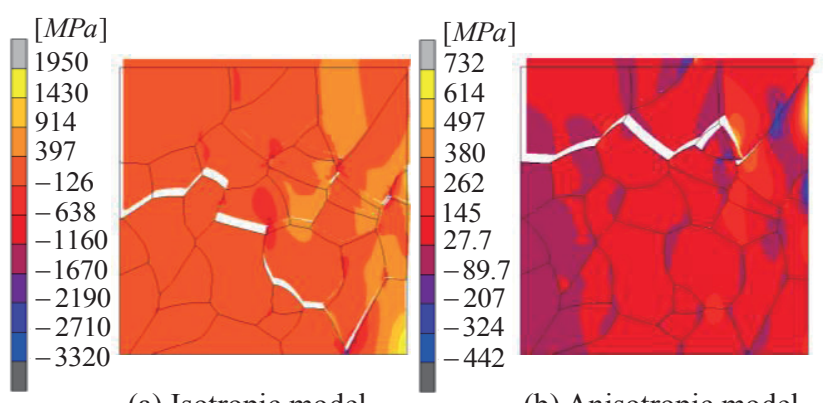

(a) Isotropic model

(b) Anisotropic model

Fig. 7 Tensile stress distributions of Model 30-1 $(A=0.01)$.

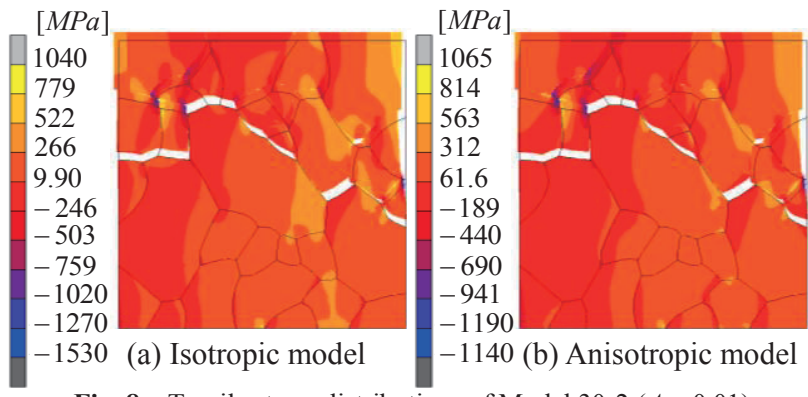

Fig. 8 Tensile stress distributions of Model 30-2 $(A=0.01)$.

\subsection{Influence of grain configuration}

As one of the possible factors to affect the microstructural fracture behavior, the grain configuration can be considered. In order to examine this effect, the fracture behaviors in Model 30-1 and 30-2 shown in Fig. 1 were compared with assuming the interaction parameter $A$ of 0.01 for clarifying the influence of parameter $A$. The computed results with both the isotropy and anisotropy in grain under applying $1.800 \%$ strain were summarized into Figs. 7 and 8 . There cannot be seen any significant difference in the results of Model 30-2, while not only the crack paths but also the tensile stress distributions in Model 30-1 were very different. Although it can be considered that the grain boundary would be easy to deform in shear direction to grain boundary, the anisotropy in grain largely affected the fracture behavior in Model 30-1. So, it was revealed that the grain configuration was one of the most important factors to examine the microstructural fracture behavior

\section{Conclusions and future works}

In order to demonstrate the microstructural fracture behavior in metal materials, the finite element method with the interface element were applied for examining the two-dimensional deformation in the models obtained through Voronoi tessellations. From the serial computations by changing elastic property in grain (isotropy or anisotropy), mechanical property at grain boundary (interaction between opening and sliding deformation), and grain configurations, it was revealed that all the factors varied in this research might affect the microstructural fracture behavior. Also, it can be concluded that the interface element can be useful 
for demonstrating the deformation at grain boundary. Moreover, it was found that not only the modification of stiffness in interface element but also the additional factors such as plasticity in grain have to be taken into account as the future works.

\section{Acknowledgements}

This work was conducted as a part of researches in an inter-university cooperative research project, Development Base of Advanced Materials Development and Integration of Novel Structured Metallic and Inorganic Materials.

\section{References}

1) H. Serizawa, C.A. Lewinsohn, M. Singh and H. Murakawa : Numerical Simulation of Fracture Behavior of Ceramic Composite Joints By Using A Combined Interface Potential, Transactions of JWRI, Vol.33, No.2 (2004), pp.181-185.

2) N. Igata : Strength of Materials, Tokyo, BAIFUKAN Co., Ltd, (1983), pp.11 (in Japanese).

3) T. Tanaka, S. Tsurekawa, H. Nakashima and H. Yoshinaga : Misorientation Dependence of Fracture-Stress and Grain-Boundary Energy in Molybdenum with [110] Symmetrical Tilt-Boundaries, Journal of the Japan Institute of Metals, Vol.58, No.4 (1994), pp.382-389 (in Japanese).

4) H. Nakashima and M. Takeuchi : Grain Boundary Energy and Structure of $\alpha-\mathrm{Fe}(110)$ Symmetric Tilt Boundary, TETSU-TOHAGANE, Vol.86, No.5 (2000), pp.357-362 (in Japanese). 